\title{
La difficile reconfiguration des fronts d'eau pour les villes menacées d'inondation : New York et sa Dryline
}

\author{
Marilyne Gaudette ${ }^{a}$, Sylvain Lefebvre ${ }^{b}$
}

RÉSUMÉ. Le passage dévastateur de l'ouragan Sandy sur la côte est américaine en octobre 2012 est apparu comme un levier pour repenser l'aménagement des berges en vue de se préparer à la récurrence inévitable d'autres tempêtes extrêmes. Ne pouvant être relevés que par l'innovation, les défis posés par les changements climatiques ont incité le gouvernement fédéral à lancer le concours international Rebuild by Design dans l'objectif de développer des stratégies adaptatives se distinguant des mesures de protection rigides habituellement préconisées. Nécessitant une évolution conséquente des modes de planification, une vaste démarche de conception intégrée impliquant les équipes finalistes et des acteurs de différents horizons a été mise en branle. Parmi les projets phares retenus figurent la Dryline et son parc éponge. Ce système de protection intégré destiné à rendre la pointe sud de l'île de Manhattan résiliente aux inondations a contribué à dessiner les contours d'une approche audacieuse basée sur une plus grande accommodation de l'eau en milieu fortement urbanisé, jusqu'à ce qu'un lot de contraintes locales enclenche un retour vers une stratégie de gestion de risques plus traditionnelle.

\begin{abstract}
The devastating passage of hurricane Sandy, on the American eastern coast in October 2012, appeared like an opportunity to redesign the shore layout in view of preparing for an inevitable recurrence of other extreme storms. Since taking up this challenge could only be done by innovation, the challenges brought on by climate changes prompted the federal government to launch the Rebuild by Design international contest, with the objective of developing adaptive strategies that distinguish themselves from the rigid protection measures commonly recommended. Since this required evolution consistent with the planning methods, a vast integrated design undertaking was launched involving finalist teams and actors from different backgrounds. Amongst the leading projects selected were Dryline and its water retention park. This integrated protection system, intended to make the southern end of Manhattan Island resilient to floods, contributed to designing the structure of an of an audacious approach. This approach is based on larger water accommodation capacity in a heavily urbanized environment, up to when a number of local constraints triggers returning to a more traditional risk. management strategy.
\end{abstract}

\section{Introduction}

New York a connu par le passé plusieurs épisodes de tempêtes extrêmes qui ont provoqué des inondations considérables à l'échelle de la ville. Sous l'effet d'un déni du danger, d'une acceptation sociale trop élevée du risque ou d'un certain fatalisme, les retours d'expériences post-catastrophes ont été néanmoins largement ignorés des programmes urbanistiques et architecturaux, jusqu’à ce qu'un évènement de grande ampleur bouleverse

\footnotetext{
a Doctorante, Département d'études urbaines et touristiques, Université du Québec à Montréal

b Professeur titulaire, Ph. D., Département de géographie, Université du Québec à Montréal
}

l'imaginaire collectif et fasse émerger de nouvelles représentations du risque.

C’est la prise de conscience générée par le passage de l'ouragan Sandy le 29 octobre 2012 qui a contribué à remettre en question la pertinence des efforts de récupération, de relèvement et de reconstruction habituellement déployés par les différentes administrations fédérales et municipales pour mettre fin aux situations de crise et pour retrouver un mode de fonctionnement acceptable. Contribuant à mettre en relief la vulnérabilité d'une ville qu'on 
pensait à l'abri des catastrophes de cette ampleur' l'ouragan Sandy a ainsi provoqué un basculement de la gestion de crise à la gestion du risque, tandis que la période post-catastrophe a ouvert une fenêtre d'opportunité à la mise en œuvre d'actions visant à modifier la trajectoire de développement et de mise en valeur du front d'eau de la métropole. La nécessité de mettre en place des mesures de prévention a, par conséquent, constitué une occasion de «faire la ville» autrement pour arriver à se préparer à la récurrence inévitable d'autres perturbations climatiques et à s'adapter à l'horizon incertain d'un environnement changeant.

Bien que la combinaison de la marée haute, de la taille de la tempête, de sa trajectoire inhabituelle et de son angle d'approche ait été exceptionnelle et catastrophique (City of New York, 2013), ses effets ont été sans équivoque le produit de la longue histoire du développement urbain de la ville. L'altération, l'artificialisation et l'expansion continues des bandes riveraines de l'île de Manhattan au cours des siècles auraient, en effet, contribué à exacerber la vulnérabilité de la ville en l'exposant davantage aux aléas climatiques. Un renversement des modes de planification et d'aménagement traditionnels a été, dans ce contexte, instauré par le gouvernement fédéral, par l'entremise de l'importation d'un savoir-faire spécifique. Les expériences pionnières menées au sein de villes de plusieurs pays détenant une expertise en matière d'inondation ont servi d'inspiration.

Washington a tout particulièrement fait appel à une expertise néerlandaise afin d'orienter et de diriger un groupe d'intervention mandaté pour réfléchir à la reconstruction des trois États sinistrés: New York, le New Jersey et le Connecticut. Ce groupe d'experts a fait la recommandation de créer un concours international de design urbain destiné à développer des solutions innovantes répondant aux vulnérabilités les plus pressantes de ces États (Rebuild by Design, 2015). Lancé en 2013, le concours Rebuild by Design s'est imposé comme un nouveau type de compétition de design urbain. Basée sur un processus de recherche collaborative et interdisciplinaire, la démarche de cocréation privilégiée, fondamentalement différente du modèle fédéral habituel en matière de reconstruction, a placé les communautés et les acteurs locaux au cœur du processus de conception. L'aspect innovant de cette démarche a d'ailleurs percolé sur les projets d'aménagement des 10 équipes finalistes.

Le cabinet Bjarke Ingels Group (BIG) ${ }^{2}$, avec son projet du $B I G U$, a été l'un des six finalistes qui se sont partagé les fonds fédéraux dans le cadre de ce concours. Visant à rendre la pointe sud de l'île de Manhattan résiliente à la montée actuelle des eaux et au risque inhérent de débordement des eaux de tempête, ce grand projet urbain incarne une infrastructure de protection intégrée épousant la morphologie de l'île sur une distance de 16 kilomètres. Initialement nommé en l'honneur du cabinet d'architectes qui en est à l'origine et de sa forme caractéristique, ce projet a, par la suite, été renommé Dryline. Si cette nouvelle appellation a été motivée pour éviter la promotion d'un seul cabinet $^{3}$, elle se voulait également un clin d'œil à la High Line, cette célèbre promenade urbaine newyorkaise aménagée sur une ligne ferroviaire surélevée désaffectée.

Bien que les villes côtières fortement exposées aux ouragans continuent de privilégier les mesures visant à repousser l'eau et à lui résister en aménageant des digues toujours plus hautes, une solution innovante aux traditionnelles infrastructures de protection rigides a été ainsi imaginée sous l'impulsion d'un nouvel idéal de ville résiliente aux inondations (Ovink et Boeijenga, 2018). Parmi les stratégies adaptatives retenues, le concept de parc éponge a assurément été le plus audacieux. Conçue afin de s'adapter avec souplesse aux éventuelles perturbations de l'environnement, cette typologie de parc permet d'absorber, de filtrer, d'emmagasiner et de réutiliser l'eau excédentaire, et représente ainsi une nouvelle façon de prévenir les inondations. Cela dit, un certain nombre de blocages au regard de l'acceptation du risque et de ses conséquences, jumelé à un lot de contraintes techniques et financières liées à l'entretien du parc, aux infrastructures de drainage des eaux et aux procédés de construction mobilisés, a contribué à entraver la traduction de ce concept à l'échelle locale, si bien que ce projet novateur s'est écarté de la vision initiale pour effectuer un retour vers une planification et un aménagement plus traditionnels.

Cet article propose de faire une description et une analyse du concours Rebuild by Design. Nous porterons un regard sur une portion particulière de 
la Dryline, soit celle destinée au remaniement du East River Park. Cette dernière étant la plus aboutie en matière de planification, nous serons à même de constater l'évolution des choix d'aménagement effectués. Pour ce faire, nous proposons d'abord de revisiter la démarche adoptée afin d'apprécier l'origine et la portée de son caractère innovant, puis d'examiner la proposition d'aménagement initiale en insistant sur les éléments de design novateurs proposés. À la suite de la présentation du concept de parc éponge, nous nous pencherons sur la traduction des plans à l'échelle locale, pour enfin comprendre la manière dont les conditions de mise en œuvre, façonnées par d'importantes contraintes locales, ont escamoté l'aspect novateur tant acclamé. Au final, des leçons pourront être tirées de la démarche du concours afin d'éviter que soient reproduites les erreurs du passé et de s'assurer qu'à l'avenir les projets d'aménagement issus d'un processus de cocréation ne soient pas relégués au plan de bonnes intentions et de vœux pieux.

\section{Rebuild by Design :} une idée novatrice pour sortir de l'impasse des risques climatiques

\subsection{La genèse de l'innovation}

L'essence du concours Rebuild by Design trouve ses racines dans le leadership et l'instinct politique du président américain de l'époque, Barack Obama. Devant le rayonnement multiscalaire et l'effet domino des répercussions des inondations côtières, le morcellement en silos étanches des différentes agences fédérales devenait efficace (logement et développement urbain, transport, énergie, etc.). Ces agences devaient au contraire être amenées à coopérer pour que l'impératif et l'urgence climatiques s'imposent dans tous les domaines de compétences.

Au lendemain du passage de l'ouragan Sandy en 2012, Obama a donné l'ordre de créer le Hurricane Sandy Rebuilding Task. Force en vue d'implanter une vision transversale au sein de l'administration, et d'aligner les politiques et les ressources du gouvernement fédéral avec les priorités et les besoins locaux. Composé de 23 agences fédérales et de 5 départements relevant de Washington, ce groupe de travail, chapeauté par le U.S. Department of Housing and Urban Development (HUD), avait pour objectifs de fournir une aide efficace et coordonnée aux communautés côtières sinistrées, de remettre en état les infrastructures essentielles endommagées (hôpitaux, routes, infrastructures de traitement des eaux usées, d'approvisionnement en eau et en énergie, etc.) et d'orchestrer la reconstruction.

Ce premier effort de décloisonnement sectoriel, en se traduisant par une coopération entre des entités habituellement segmentées et autonomes, a mené à l'adoption d'une prise de position audacieuse. Plutôt que de reproduire les erreurs du passé en reconstruisant les infrastructures et le cadre bâti à l'identique, les différents représentants fédéraux ont fait le pari de reconstruire autrement afin de s'assurer que la région ne subisse pas trop de ravages lors des prochaines tempêtes et puisse rebondir adéquatement dans une telle éventualité (Ovink et Boeijenga, 2018).

Le désir de mettre à profit un savoir-faire reconnu à l'échelle mondiale en matière de prévention des inondations a donné lieu à une prise de contact avec le gouvernement néerlandais. Lors d'une visite exploratoire aux Pays-Bas, Shawn Donovan, secrétaire du HUD et président du Task Force, a proposé à Henk Ovink, spécialiste de la gestion de risques liés à l'eau, de piloter le groupe de travail sur la reconstruction. Cette entente s'est traduite par la poursuite d'une ambition commune : faire de la région de New York, du New Jersey et du Connecticut une vitrine de la résilience côtière. Exigeant de sortir des sentiers battus, cette ambition a nécessité de développer une approche foncièrement différente des procédures fédérales habituelles en matière de reconstruction et, ainsi, de s'inscrire dans la continuité de ce que le président avait mis de l'avant depuis l'automne 2012 (Ovink et Boeijenga, 2018).

Inspirée de la tradition néerlandaise en matière de démarche de planification et de design intégrés ${ }^{4}$, l'approche envisagée devait favoriser et encadrer la collaboration intensive d'une multitude de parties prenantes pour ainsi permettre certaines formes d'émulation et d'itération. Lentement, l'idée d'un concours de design collaboratif et inclusif a germé. Si les concours représentent un moyen d'obtenir un éventail de solutions innovantes et créatives à un problème donné par leur capacité à rassembler différentes professions et perspectives d'analyse, le concours dont il était question devait également favoriser l'implication des communautés locales 
dans l'élaboration de scénarios de reconstruction. Le modèle usuel de la compétition a ainsi été repensé afin de permettre aux chercheurs, aux élus locaux, aux professionnels et aux groupes communautaires de travailler, d'expérimenter, d'apprendre et d'innover ensemble. Incubatrice d'innovations et de créativité, cette approche intégrée a favorisé une démarche itérative favorable à l'adoption de projets porteurs bien ancrés dans la réalité locale. Le concours a été nommé Rebuild by Design (Ovink et Boeijenga, 2018).

Progressive et dynamique, la grande région de New York constituait un laboratoire fertile pour expérimenter une telle démarche. Le principal défi résidait néanmoins dans la nécessité de la faire entrer dans les paramètres des cadres législatifs et institutionnels américains. Si l'America Creating Opportunities to Meaningfully Promote Excellence in Technology, Education, and Science Act (abrégée sous l'appellation America COMPETES ACT) octroyait au HUD le pouvoir d'administrer le concours (HUD, 2014), la rigidité des procédures en place posait néanmoins certains problèmes.

Traditionnellement, après une catastrophe, le gouvernement fédéral offre une aide aux États et aux gouvernements locaux en leur accordant des allocations basées sur l'estimation des dommages causés par le désastre. Les États et villes sont alors invités à définir leurs priorités et à présenter des projets de reconstruction à chacun des départements et agences concernés afin de se qualifier et de mettre la main sur ces fonds. Dans le contexte de l'ouragan Sandy, 60 G\$ US en fonds fédéraux allaient être alloués à la réparation des infrastructures et répartis entre diverses agences gouvernementales (Ovink et Boeijenga, 2018) Une refonte du système fédéral de financement visant à restreindre la fragmentation des fonds - et, par conséquent, la segmentation des efforts de redressement - demeurait malgré tout essentielle pour donner préséance à des interventions concertées plus novatrices. De plus, à défaut de pouvoir imposer les projets, le gouvernement fédéral souhaitait stratégiquement faire adhérer les gouverneurs et les maires aux projets en favorisant des solutions en adéquation avec les besoins et les priorités locales pour, en définitive, encourager leur matérialisation (Ovink et Boeijenga, 2018).
Malgré une forte résistance politique contre l'idée de limiter le montant des subventions destiné à répondre aux besoins immédiats des sinistrés pour financer un concours de design dont la portée et les retombées demeuraient incertaines, le Congrès est allé de l'avant en faisant le pari que les projets retenus allaient contribuer à tester un certain nombre de stratégies d'aménagement originales qui, une fois mises sur pied, seraient susceptibles d'insuffler des initiatives semblables qui seraient à l'origine d'un réel changement de culture en matière de gestion des risques d'inondation (Rebuild by Design, 2015).

En juin 2013, le concours a été intégré au Climate Action Plan lancé par la Maison-Blanche. Un montant de 1 G\$ US en fonds fédéraux allait être alloué aux États et aux villes par le biais de la Community Development Block Grant for Disaster Recovery (CDBGDR) pour la mise en œuvre des projets (HUD, 2014). Nonobstant l'obtention de ces fonds, $4 \mathrm{M} \$$ supplémentaires étaient nécessaires pour la tenue du concours. Ce sont des dons philanthropiques obtenus grâce au soutien de 10 partenaires financiers $^{5}$ qui ont permis de le mener à terme. Le concours a été officiellement ouvert le 20 juin 2013 (Ovink et Boeijenga, 2018).

\subsection{La démarche des finalises du concours}

Le concours a été divisé en plusieurs étapes. Tout d'abord, un appel d'offres invitant des équipes multidisciplinaires du monde entier à articuler leur approche de la résilience côtière a été publié ${ }^{6}$. Ainsi, 148 équipes de concepteurs en provenance de 15 pays ont soumis leur candidature. Du nombre, 10 équipes finalistes ont été retenues par le jury afin d'entamer une démarche concertée de planification et de conception dans différents secteurs des États de New York, du New Jersey et du Connecticut (Rebuild by Design, 2015). Cette première ronde a mené au lancement d'une importante phase de recherche collaborative.

Durant trois mois, les équipes finalistes ont sillonné près de 30 communautés côtières afin de comprendre les réalités locales, de côtoyer au quotidien leur population et, plus spécifiquement, d'interroger les sinistrés en vue de saisir leurs besoins. Différentes activités ont été élaborées afin de créer un environnement favorable aux échanges et de permettre aux participants de 
s'engager activement dans l'élaboration de pistes de recherche. Des tables rondes avec les résidents et les représentants des autorités locales de chacune des communautés touchées ont été tenues afin de dresser le portrait global des effets de tempête et des problèmes de longue date que ces derniers ont mis au jour ou exacerbés.

Plus précisément, les équipes ont pris part à une série de conférences et d'ateliers menés conjointement avec des intervenants locaux afin de mieux saisir les enjeux en matière de logement, d'infrastructures, d'économie, de santé publique, d'accès aux services publics et d'environnement. Pour élargir davantage la portée des activités de recherche, un groupe d'experts issus de diverses disciplines universitaires (urbanisme, architecture, ingénierie, sciences de la Terre, sociologie, droit, etc.) a été formé pour accompagner les équipes finalistes du concours dans leurs réflexions. Détenant des expertises et des compétences complémentaires, les équipes ont rapidement été amenées à partager et à mettre en commun leurs données et leurs observations. À terme, cet effort d'apprentissage et d'analyse collectif et incrémental - un exercice habituellement absent d'un contexte de compétition - a permis d'enrichir considérablement le diagnostic territorial des vulnérabilités vis-à-vis des phénomènes climatiques extrêmes à l'échelle régionale. Ce diagnostic a conduit en retour à l'identification de leviers d'action potentiels et d'occasions de transformation, qui ont mené ultimement à la formulation de concepts d'aménagement préliminaires (Rebuild by Design, 2015).

En octobre 2013, 41 projets répondant aux défis identifiés précédemment ont été dévoilés aux responsables locaux, aux représentants fédéraux, à la presse ainsi qu'à plus de 1000 individus issus du grand public. Un concept d'intervention par équipe finaliste a ensuite été sélectionné par le HUD en fonction d'une série de critères très précis. Outre le mérite technique et l'ancrage local des concepts, des considérations d'ordre politique sont également venues teinter le processus de sélection, dans la mesure où la capacité des communautés à mener à terme les projets a été une variable déterminante. Enfin, 10 approches visionnaires visant à rendre la région plus résiliente aux risques climatiques ont été retenues, donnant ainsi le feu vert à la dernière étape du concours : la conception des projets.

Revue Organisations \& Territoires $•$ Volume $28 \cdot$ № $2 \cdot 2019$
Parmi les sites new-yorkais potentiels identifiés par l'équipe du cabinet BIG (Manhattan, Red Hook et South Bronx), la proposition visant à reconfigurer la pointe sud de l'île de Manhattan était la plus prometteuse. Une démarche de réflexion et de conception itérative habituellement réservée aux cabinets privés a été entamée dans le but de définir une vision intégrée de développement et d'élaborer collectivement une solution novatrice vouée à renforcer la résilience aux inondations du littoral de l'île. Plusieurs activités de codesign ont été proposées à ce titre.

Au cours des premiers ateliers, 26 groupes communautaires du Lower East Side, des acteurs issus du milieu des affaires et des élus locaux ont débattu de la portée et des limites de diverses approches en matière d'adaptation aux inondations de manière à mettre en évidence les stratégies pouvant potentiellement être mises en pratique. Les résultats de ces discussions ont débouché sur l'élaboration de deux prototypes d'aménagement présentés sous forme de modèles interactifs dans la deuxième série d'ateliers. Destinés à guider et à faciliter les discussions, ces modèles ont ensuite été soumis à une analyse critique dans l'objectif de réajuster, d'affiner et de bonifier les propositions. Les différentes parties prenantes ont d'ailleurs eu l'occasion d'expérimenter un certain nombre de stratégies en construisant leur front d'eau à l'aide de modules en mousse recréant des bermes naturelles, des murs de protection et des équipements publics. À terme, cette phase de conception itérative a été l'occasion de rallier les groupes d'intérêts divergents à une cause commune, soit la résilience côtière, et de construire des coalitions politiques et communautaires ayant joué un rôle de premier plan dans la mise en œuvre du projet (Ovink et Boeijenga, 2018).

\subsection{L'évaluation des propositions finales}

Après des mois d'engagement communautaire et de conception itérative, les 10 équipes finalistes ont complété leur scénario d'aménagement, exposé leur maquette au cours d'une exposition publique et présenté leur proposition aux membres d'un jury multidisciplinaire composé d'experts issus des milieux professionnel et universitaire? ${ }^{7}$. Si leur mission consistait à identifier les propositions susceptibles de répondre aux normes les plus élevées en matière de conception, d'innovation et de résilience côtière, d'autres critères d'évaluation plus spécifiques leur ont été fournis : 
ampleur du processus de recherche et d'analyse, démonstration du processus participatif et des coalitions formées, qualité des solutions proposées et pertinence de la stratégie de mise en œuvre. Les délibérations ont été, quant à elles, accompagnées de commentaires émanant d'agences fédérales et d'organes du gouvernement local susceptibles d'être responsables de la mise en œuvre des concepts gagnants. À la suite de l'examen minutieux des 10 propositions, les membres du jury ont formulé des observations, des idées et des recommandations qui ont servi de base à la sélection des propositions gagnantes (Rebuild by Design, 2015).

En juin 2014, les six projets les plus prometteurs et novateurs en matière de résilience côtière ont été annoncés lors d'une conférence de presse à laquelle ont assisté le gouverneur de New York Andrew Cuomo, le gouverneur du New Jersey Chris Christie et le maire de la Ville de New York Bill de Blasio. Hormis la Dryline du cabinet BIG, les projets Living with the Bay (Interboro), Hunts Point L ifelines (PennDesign/OLIN), Living Breakwaters (SCAPE/Landscape Architecture), New Meadowlands (MIT CAU + ZUS + URBANISTEN) et Resist, Delay, Store, Discharge (OMA), respectivement développés pour les arrondissements du Queens, du Bronx et de Staten Island à New York ainsi que de Teterboro et Hoboken au New Jersey, ont été sélectionnés. Une somme de 930 M\$ US en fonds fédéraux a ainsi été allouée à la Ville de New York ainsi qu'aux États de New York et du New Jersey afin de mettre en œuvre ces projets ${ }^{8}$. Du montant, $335 \mathrm{M}$ \$ ont été octroyés pour le BIG U (HUD, 2014). La réalité politique a d'ailleurs été partie intégrante des délibérations et a influencé la distribution des fonds. En effet, plus le soutien public était important, plus il devenait intéressant pour le gouvernement fédéral de financer le projet dans l'optique de faciliter sa mise en œuvre, dans le respect du concept développé (Ovink et Boeijenga, 2018).

Certaines exigences préalables à l'octroi des fonds étaient néanmoins imposées aux États et aux administrations locales par le HUD. Parmi celles-ci figurait la nécessité d'incorporer les projets issus du concours dans un plan d'action plus large, soit le Disaster Recovery Action Plan. Ce document de planification devait permettre au HUD d'exercer un droit de regard sur la manière dont les fonds seraient mobilisés pour le relèvement à long terme, pour la remise à niveau des infrastructures et du cadre bâti et pour la revitalisation économique des zones sinistrées, mais aussi sur les stratégies retenues pour développer les propositions d'aménagement et pour assurer l'implication continue des communautés locales (HUD, 2014; Rebuild by Design, 2015). Des conditions supplémentaires ont, dans certains cas, été soumises. La Ville de New York devait notamment assurer qu'une portion de son allocation satisfaisait les besoins de la New York City Housing Authority (NYCHA) - un organisme public de l'administration de New York qui gère les logements sociaux et les habitations à loyer modique des cinq arrondissements - en matière de rétablissement post-catastrophe et de résilience côtière. Puisque la concentration d'habitations sociales est particulièrement intense le long de la East River, la Ville de New York a identifié ce secteur comme étant prioritaire et stratégique étant donné que ces populations sont souvent plus vulnérables, puis a décidé d'utiliser les fonds fédéraux pour financer la mise en œuvre d'une section de la Dryline consacrée au remaniement du East River Park9 (HUD, 2014). Les plans d'action dûment complétés, le HUD examinerait et approuverait les documents avant que les fonds ne soient versés pour l'avant-projet (planification, design, études et analyses préliminaires, etc.) et pour la construction elle-même. Les bénéficiaires ont jusqu'au 30 septembre 2022 pour utiliser le financement (Rebuild by Design, 2015). En somme, ces plans d'action se veulent un guide pour assurer les phases subséquentes de mise en œuvre des projets finalistes. Ce sont là les dernières étapes du processus par lequel le gouvernement fédéral, par l'entremise de Rebuild by Design, s'est inséré comme jamais auparavant dans les décisions normalement laissées aux États et aux gouvernements locaux (Ovink et Boeijenga, 2018).

\section{La vision initiale du cabinet BIG}

L'ambition de départ de l'équipe du cabinet d'architecture BIG a été d'introduire un dispositif de protection contre les inondations sur le front d'eau de Manhattan en évitant de créer une barrière et une séparation entre la ville et l'eau. Un exercice d'émulation réalisé à partir de la réinterprétation d'une infrastructure existante et de l'exploitation d'un pan de l'histoire de la ville allait à ce titre poser les jalons de ce qui allait devenir un système de protection intégré de 15 kilomètres de long. Les leçons tirées de la High Line ont constitué dans un premier temps une inspiration centrale. À la manière de cette ligne ferroviaire aérienne reconvertie en promenade urbaine, la Dryline allait conjuguer infrastructures et équipements de loisir, cette fois de manière simultanée. Le caractère 
multifonctionnel de cette infrastructure permettrait d'assurer la protection des individus, tout en accommodant des activités récréatives quotidiennes grâce à une programmation riche et diversifiée (Rebuild by Design, 2015). Au-delà de sa visée fonctionnelle, la Dryline allait participer à réactiver l'espace public et devenir un levier de transformation positive de l'espace (Ovink et Boeijenga, 2018).

Deux figures emblématiques du développement moderne de la ville de New York ont également été exploitées dans le concept d'aménagement de la Dryline. L'équipe du cabinet d'architecture BIG avait en effet pour ambition d'évoquer le choc d'idéologies à l'origine de luttes historiques en matière de planification en mariant de manière habile les idées de l'urbaniste Robert Moses (1888-1981) et la vision à l'échelle humaine de l'auteure et activiste de la cause urbaine Jane Jacobs (1916-2006) ${ }^{10}$. Nécessitant une approche globale et à grande échelle, cette infrastructure de protection colossale allait néanmoins embrasser les quartiers avoisinants. Unifiée dans sa fonction, cette infrastructure de protection à géométrie variable allait être intégrée au tissu urbain existant, et répondre aux besoins et aux désirs des communautés locales (Rebuild by Design, 2015).

La mise sur pied d'une série de mesures de protection adaptatives allant de la 57e Rue à l'ouest, en passant par The Battery au sud, jusqu'à la 42e Rue à l'est caractérisait le concept d'aménagement retenu. Destinée à protéger la pointe sud de Manhattan avec des aménagements plus adaptés à la montée actuelle des eaux et au risque inhérent de débordement des eaux advenant une forte tempête, cette proposition finale était composée de trois principaux « compartiments $»^{11}$ imaginés et conçus au regard des caractéristiques des différents quartiers visés par le projet et des enjeux qui s'y dessinaient. En formant une trame continue, ces compartiments demeuraient néanmoins indépendants et étaient suffisamment flexibles pour qu'on les développe de manière subséquente dans le temps (Rebuild by Design, 2015).

Bien que le design des trois compartiments ait fait l'objet de stratégies d'aménagement spécifiques de la part de l'équipe du cabinet d'architecture BIG, un seul d'entre eux a jusqu'ici fait l'objet d'une planification approfondie à l'échelle locale, soit la transformation du East River Park.

\subsection{La transformation \\ du East River Park en parc éponge d'envergure}

Localisé au cœur d'un vaste chapelet d'espaces publics et d'équipements sportifs de proximité et adossé à une série de voies rapides isolées, ce compartiment proposait d'ériger un mur de soutènement en façade des voies de circulation existantes, sur lequel reposerait un talus vallonné descendant en pente douce vers la East River. Grâce à son relief topographique, ce talus incliné permettrait de protéger la ville de l'envahissement des eaux lors d'épisodes d'inondation, tandis que le parc en lui-même favoriserait la rétention des eaux au moyen de plantations résistantes et tolérantes à l'eau salée. Inondable et perméable, ce parc éponge permettrait à la rivière de fluctuer naturellement et de tirer profit des services écosystémiques offerts par la nature. Selon une architecte paysagiste du cabinet BIG, des objectifs de mitigation et d'adaptation étaient par conséquent atteints (échange téléphonique, 13 mars 2019). Afin d'assurer la liaison entre le parc et les différents quartiers adjacents, d'importants ponts piétonniers paysagers recouvriraient ponctuellement la Franklin D. Roosevelt Drive (F.D.R. Drive). Contoumant habilement les infrastructures sportives existantes, le talus maintiendrait les usages récréosportifs actuels, tout en offrant de nouvelles activités.

S'inscrivant en continuité des stratégies d'adaptation préconisées aux Pays-Bas, le talus paysagé serait ainsi le premier maillon d'une série de compartiments encerclant la pointe sud de l'île de Manhattan ${ }^{12}$. La nature audacieuse de ce concept de parc résilient aux inondations s'est néanmoins heurtée à une réalité brutale. Une série de contraintes techniques, financières et de temps sont venues altérer la vision initiale du concept et le programme d'aménagement, tant et si bien qu'un basculement idéologique s'est effectué. La logique d'accommodation de l'eau, initialement omniprésente au sein du concept de parc éponge, a lentement dérivé vers une logique de protection plus traditionnelle.

\section{L'atterrissage des plans à l'échelle locale : l'amorce d'un changement idéologique majeur}

La fin du concours a marqué le démarrage d'une démarche de planification concertée du premier segment d'intervention financé de la Dyyline qui, à terme, 
mènerait à la mise en œuvre de ce qu'on connait aujourd'hui sous le nom de East Side Coastal Resiliency Project. Ce passage de l'étape «concept» à l'étape "projet» a pour objectif de traduire la vision et le concept d'aménagement développés par le cabinet BIG à l'échelle locale en les mettant en présence des contraintes inhérentes au terrain. Parallèlement au peaufinage du design en collaboration étroite avec les communautés locales, une série d'études techniques préliminaires ont à cette étape été produites par différents départements de la Ville (tests de sol, étude géotechnique, inspection des infrastructures souterraines et des ponts piétonniers, étude de circulation piétonnière, inventaire des arbres, etc.) en vue des processus d'approbation de différentes agences fédérales, étatiques et municipales et, ultimement, de la délivrance du permis de construction (HUD, 2014). Certains éléments ont, à ce titre, été remis en question, puis reconsidérés.

D'entrée de jeu, la relation entre les idéaux véhiculés par le concept de parc éponge et la réalité terrain a fait surgir un certain nombre d'interrogations, notamment en raison de la nature apparemment utopique, pour certains, du projet. Une question centrale en lien avec le rôle des parcs dans la ville est notamment venue à l'esprit de certains représentants municipaux : «Est-ce que les parcs sont utilisés pour protéger le quartier ou ont-ils eux-mêmes besoin de protection? » (Ovink et Boeijenga, 2018, p. 230, trad. libre) Cette nouvelle typologie de parcs proposait une approche jusqu'ici absente de l'infrastructure actuelle de parcs et d'espaces verts de la ville. L'inondation temporaire du East River Park imposait, une fois les eaux retirées, un nettoyage en profondeur des installations sportives et des équipements en place ainsi qu'un temps de latence afin de permettre à la nature de se rétablir et de retrouver ses conditions initiales (échange téléphonique, 13 mars 2019). Plus encore, cette approche supposait d'accepter le risque d'inondation et de vivre avec les dynamiques naturelles, leurs incertitudes et leur imprévisibilité, et nécessitait donc une certaine ouverture de la part des départements responsables de l'entretien et de la construction du parc ${ }^{13}$. Par ailleurs, contrairement aux parcs passifs qui font office de plaines inondables, le East River Park dispose d'installations sportives, d'éclairage et de gazon synthétique non adaptés aux inondations, ce qui rend le projet encore plus difficile à réaliser (Silver, 2019).

Le concept de parc éponge posait également un nombre important de défis techniques, notamment en ce qui a trait au drainage des eaux. Bien que le mur de soutènement sur lequel prendrait appui le talus incliné permettrait de garder la ville au sec, ce dernier, en créant une ceinture hermétique en bordure des quartiers, empêcherait le ruissellement de l'eau vers la East River. Si l'idée de rendre, à moyen terme, les quartiers adjacents perméables à l'eau a été sérieusement considérée (échange téléphonique, 13 mars 2019), deux types d'infrastructures souterraines destinées à gérer les eaux de pluie et les eaux de tempête devaient entre-temps être intégrés aux plans détaillés : d'une part, la mise en place de réservoirs de rétention, de pompage et de stockage temporaire des eaux localisés sous le parc et, d'autre part, l'aménagement d'un système d'adduction d'eau parallèle à la F.D.R. Drive activé lors d'épisodes d'inondation visant à réacheminer les eaux vers deux intercepteurs. Dans les deux cas, l'eau serait libérée vers la rivière à la suite de son retrait et de la stabilisation du réseau d'égouts (NYC Mayor Office of Recovery and Resiliency, 2017, 2018a).

La construction du mur de soutènement le long de la F.D.R. Drive posait également d'autres défis majeurs. L'enfoncement des pieux devait, dans un premier temps, être réalisé à proximité du lieu de vie de 15000 résidents du Lower East Side et causerait des nuisances importantes aux riverains. La construction engendrerait aussi la fermeture d'une voie en soirée et au cours de la nuit pour accommoder l'équipement nécessaire aux travaux, ce qui perturberait la circulation. Finalement, la construction du mur de protection impliquerait l'excavation complète d'une ligne de transmission provenant du système électrique central ConEdison, puis la construction d'un tunnel de protection massif autour de cette infrastructure souterraine. La nécessité de la garder active poserait de sérieux défis techniques, tout en étant une intervention extrêmement coûteuse (échange téléphonique, 13 mars 2019). En somme, l'ensemble de ces contraintes aurait une incidence majeure sur la capacité à mener à terme la transformation du East River Park en parc éponge (Grillo, 2019).

Malgré l'avancement considérable des documents de planification et l'enthousiasme médiatique entourant le concept de parc éponge, la diffusion d'un communiqué de presse en septembre 2018 a contribué à faire basculer la transformation du East River Park. Après quatre années de concertation entre l'équipe du cabinet BIG, les différents départements de la Ville et les groupes communautaires, ce concept a abouti à une 
fin de non-recevoir de la part de l'administration municipale, et ce, au grand dam des acteurs ayant pris part à la planification méticuleuse du projet. On a plutôt choisi de faire place à une approche plus traditionnelle en matière de gestion des risques axée sur une logique de résistance et de protection complète contre les inondations. Au lieu d'accommoder la présence potentielle de l'eau au sein du parc lors d'épisodes d'inondation, l'idée de rehausser le parc de trois mètres au-dessus du niveau de la mer s'est finalement imposée au sein de l'administration municipale. Ce changement de concept délocalise la mesure de protection, initialement adossée à la F.D.R. Drive, vers la limite du front d'eau (NYC Mayor Office of Recovery and Resiliency, 2018b).

Les motifs de ce changement soudain d'orientation sont nombreux et intimement liés aux défis et contraintes énumérés précédemment. Dans un premier temps, le nouveau concept de parc surélevé, en garantissant l'exclusion de tous les équipements sportifs et du mobilier urbain hors de la zone inondable, rendra caduc le budget d'entretien qui devait initialement être alloué pour la restauration du parc à la suite d'un évènement d'inondation majeur (Silver, 2019). Dans un second temps, le déplacement du chantier de construction vers la limite du front d'eau préviendrait la fermeture partielle de la F.D.R. Drive, éviterait les embouteillages générés par le camionnage grâce à l'introduction des matériaux de construction par bateaux et évacuerait les risques liés aux travaux de construction autour du système électrique central. Finalement, ce nouveau concept contribuerait à réduire les délais et l'échéancier de construction d'un an. Achevée en 2022, la mise en œuvre du nouveau plan permettra à la Ville de conserver le financement fédéral (Grillo, 2019; Hanania, 2019). Cela dit, ce projet révisé, en entraînant la reconstruction complète du parc, de l'esplanade et des équipements et terrains de sport dont certains ont récemment été rénovés -, engendrera des coûts de 1,45 G\$ US, soit plus ou moins $500 \mathrm{M} \$$ de plus que ce qui était initialement projeté (Grillo, 2019).

\subsection{Les contraintes du terrain comme barrières à l'innovation}

Bien que le concept de parc surélevé incarne bel et bien une stratégie d'adaptation aux impacts des changements climatiques, force est de constater que le caractère innovant que présentait le concept d'aménagement de parc éponge s'est, en cours de route, effrité. Initialement structuré et modelé en fonction de la East River, le concept de parc éponge aurait permis de composer avec l'eau en cas de phénomènes météorologiques sévères, plutôt que de la combattre. En favorisant l'infiltration naturelle de l'eau, cette stratégie aurait permis une atténuation du risque, à défaut de lui résister. Cette zone tampon aurait contribué à créer une interface entre l'environnement bâti dense et le milieu aquatique, et à repenser les rapports entre la ville et l'eau. La flexibilité et l'adaptabilité du parc étaient en soi uniques.

À l'inverse, le concept d'aménagement retenu se veut la réinterprétation d'une stratégie d'adaptation somme toute plus traditionnelle. L'eau n'est plus autorisée à pénétrer périodiquement dans le parc et ce dernier retrouve ainsi une fonction plus usuelle. En clair, ce concept de parc plus traditionnel, en servant de rempart contre les inondations, fera front à l'eau ${ }^{14}$ et évacuera donc la dimension performative du paysage, qui permettait d'atténuer les inondations. Contrairement au discours d'accommodation de l'eau, le retour en force de la rhétorique de protection au sein du nouveau concept d'aménagement ne contribue en effet qu'au transfert du risque dans l'espace et à l'augmentation de la vulnérabilité des secteurs non protégés adjacents.

Par ailleurs, le caractère innovant du processus collaboratif de cocréation du projet est également escamoté. D'un point de vue procédural, on assiste au retour d'une démarche et d'une structure décisionnelle typiquement descendante (top-down). Contrairement à l'approche préconisée par le concours Rebuild by Design, cette démarche écarte la prise en compte des idées des parties prenantes, au profit de l'imposition d'une vision d'aménagement défendue par l'administration municipale en place.

Les groupes communautaires impliqués dans cette démarche se sont à ce titre montrés particulièrement contrariés de ce revirement de situation. Une élue du East Village souligne que « le travail consciencieux de la communauté depuis quatre ans a complètement été mis de côté. Le nouveau plan représente un abandon radical de tout ce que la Ville avait discuté » (Hanania, 2019, trad. libre). En somme, les contraintes techniques et financières ont contribué à tempérer l'aspect novateur qu'incarnait l’idée de parc éponge, mais, plus encore, l'aspect innovant et audacieux du concours de design et de la planification concertée qui en est à l'origine. 


\section{Conclusion}

Le concours Rebuild by Design constitue un modèle innovant pour les villes menacées d'inondation qui nourrissent l'ambition de reconfigurer durablement leur front d'eau. Brisant le moule des concours de design traditionnels, le concours, lancé à New York en 2013 pour rendre les berges résilientes aux inondations, s'est éloigné de l'approche concurrentielle des compétitions et des étapes de réalisation traditionnelles habituellement préconisées. Il a favorisé la mise sur pied d'une démarche collaborative élargie moins définie par la présence de concurrents que par un réseau de participants, en plaçant les communautés au cœur du processus de conception et en introduisant une large phase de recherche préliminaire multidisciplinaire en amont de la production de concepts et de scénarios d'aménagement. De plus, la structure organisationnelle flexible du concours, extérieure aux balises de l'héritage bureaucratique fédéral et aux modes de financement traditionnels, a défié les structures formelles du pouvoir en brisant les silos institutionnels :

Rebuild by Design ne visait pas simplement les meilleurs designs - ni la réalisation de ces designs. Il visait à changer la culture, l'approche. Dès le départ, il était engagé à morceler les silos afin de changer la façon dont les gouvernements perçoivent la résilience côtière (Ovink et Boeijenga, 2018, p. 13, trad. libre).

Véritable levier de transformation, le concours a permis de réinventer la manière de trouver des solutions aux problèmes climatiques actuels et de faire une évaluation prospective des risques afin de rendre les villes côtières plus résilientes aux effets des changements climatiques. Les propositions visionnaires et innovantes issues du concours ont posé les jalons d'une réflexion renouvelée sur la résilience côtière des villes. À l'interface de la science et de la politique, du monde réel et de l'imaginaire, Rebuild by Design avait pour ambition de repousser les limites des efforts habituellement déployés en matière de reconstruction, pour ainsi ouvrir un monde de possibilités grâce à la collaboration d'un large panel d'acteurs et à l'exploitation de la créativité des professionnels.

Au final, étant donné les récents changements effectués au scénario d'aménagement initial, ce modèle de compétition expérimental était-il trop ambitieux, voire utopique? Un rapport d'évaluation réalisé par le Urban Institute (2014) ${ }^{15}$ déclarait que, malgré les bonnes intentions du concours, ce dernier demeurait, selon certains experts, plus ambitieux que réellement opérationnel. L'absence de balises, d'objectifs et de lignes directrices clairs aurait inévitablement mené à certains problèmes lors de la mise en œuvre des projets retenus par le concours, plus particulièrement celui visant la transformation du East River Park en parc éponge, et ce, nonobstant le fait que les responsables du concours aient veillé à la faisabilité globale des scénarios d'aménagement sur les plans technique, financier et politique. Les responsables auraient ainsi gagné à nouer des liens plus profonds avec les différents départements municipaux afin de s'assurer de l'ancrage local des propositions d'aménagement et de considérer les contraintes des sites.

Le Urban Institute a également recommandé qu'audelà de la conclusion d'accords de financement, des sommes supplémentaires soient octroyées pendant la mise en œuvre des projets pour assurer la concrétisation des concepts d'aménagement novateurs imaginés. En somme, bien que le concours ne se soit pas suffisamment soucié des conditions de réalisation des projets, ce dernier demeure certainement un idéal en devenir.

Un des aspects positifs de Rebuild by Design est qu'il a commencé à influencer et à transformer la manière dont les efforts de reconstruction et de prévention sont conçus, financés et mis en œuvre, non seulement à l'échelle des États-Unis, mais aussi sur la scène internationale. Le concours, qui a été largement médiatisé, a semble-t-il servi d'inspiration pour concevoir les villes côtières résilientes de demain. Aux États-Unis, le président Obama avait lancé le concours national sur la résilience aux catastrophes (la National Disaster Resilience Competition) en juin 2014, puis le concours Resilient by Design a émergé dans la région de la baie de San Francisco en 2017 (Berg, 2017). À l'échelle internationale, les responsables du concours travaillent de concert avec une douzaine de pays afin de mobiliser la démarche collaborative dans la recherche de solutions créatives à une série de problèmes urbains et environnementaux (Rebuild by Design, 2019). Dans le contexte des inondations printanières vécues au Québec et dans le reste du Canada ces dernières années, il serait à coup sûr intéressant d'importer ce modèle de concours afin d'entamer une réflexion sur l'avenir des rivages et de réaménager l'interface sensible entre ville et eau. 


\section{NOTES}

1 L'ouragan Sandy était un évènement à récurrence de 500 ans et qui avait donc 0,2 \% de risque de se produire en 2012. Au total, $17 \%$ de la superficie de la ville a été inondée.

2 Basé à Copenhague et à New York, ce cabinet est dirigé par le Danois Bjarke Ingels, une étoile montante de l’architecture.

3 L'équipe comptait en effet huit autres consultants dans les domaines de l'ingénierie, de l'architecture de paysage, de la planification urbaine, des services écologiques et de la culture (Rebuild by Design, 2015; Wainwright, 2015).

4 Un certain nombre d'expériences telles que la Biennale internationale d'architecture de Rotterdam ainsi que les programmes Room for the River et Delta font office d'inspirations et de démarches de planification exemplaires sur la scène mondiale (Rebuild by Design, 2015).

5 La Rockefeller Foundation, la JPB Foundation, la Deutsche Bank Americas Foundation, la Surdna Foundation, la Hearst Foundation, le New Jersey Recovery Fund, le NYU Institute for Public Knowledge, la Municipal Art Society, la Regional Plan Association et le Van Alen Institute.

6 L'idée étant de cibler les expertises avant de sélectionner des solutions innovantes, un appel de qualifications a été publié, au lieu du traditionnel appel d'offres.

7 Les équipes ont été encouragées à inviter des collaborateurs clés - membres de leurs coalitions communautaires et politiques, universitaires, etc. - à participer aux présentations et à démontrer leur soutien aux projets qu'ils avaient cocréés.

8 Un montant a également été alloué au projet Resilient Bridgeport (WB Unabridged/Yale Arcadis) pour poursuivre les recherches dans le Connecticut et mettre sur pied un projet pilote.

9 Les fonds fédéraux n'étaient pas suffisants pour la mise en œuvre complète de l'ensemble des projets.

10 Les grands travaux d'infrastructures et les opérations de rénovation urbaine de New York défendus et menés par l’urbaniste Robert Moses (1888-1981) au cours des années 1950 et 1960 ont été l'objet de vives contestations. Sous l'influence de Jane Jacobs (1916-2006) et d'une mobilisation citoyenne soutenue, plusieurs projets ont été tablettés en faveur d'un développement à l'échelle plus humaine. Les affronts historiques entre cet urbaniste et cette militante sont d'ailleurs souvent symbolisés par le combat de David contre Goliath (Flint, 2011; Wainwright, 2017).

11 Le terme « compartiment » est employé par l'équipe du cabinet BIG pour faire référence aux différentes sections du BIG U. Évoquant le principe d'une coque de navire, les différents compartiments de ce système de protection intégré devaient assurer l'étanchéité de la ville, malgré la défaillance ou la rupture de l'une de ses parties.

12 Le compartiment Two Bridges, localisé entre les ponts de Manhattan et de Brooklyn, incarnerait une série de murs rétractables peints par les artistes locaux et attachés sous le tablier de l'autoroute surélevée et du mobilier urbain servant de brise-lame. Le compartiment Battery Park, localisé au sud de l'île, prévoyait, quant à lui, l'aménagement d'une berme naturelle et la transformation de l'édifice de la garde côtière en « aquarium inversé » destiné à sensibiliser les populations locales et les visiteurs aux variations du niveau de la mer, tout en fournissant une barrière de protection.

13 Soit le New York. City Department of Parks and Recreation et le New York City Department of Design and Construction.

14 L'abaissement ponctuel du parc par la mise en place de grands emmarchements permettra néanmoins aux usagers de se rapprocher de l'eau (échange téléphonique, 13 mars 2019).

15 Fondé en 1968, le Urban Institute est un groupe de recherche détenant une expertise en matière de politiques sociales et économiques.

\section{RÉFÉRENCES}

Berg, N. (2017, 18 janvier). How a design competition changed the US approach to disaster response, The Guardian. Repéré à www.theguardian.com/cities/2017/jan/18/rebuild-by-design-competition-disaster-respone-climate-change

City of New York. (2013). A stronger, more resilient New York (document officiel). New York, NY: NYCEDC.

Department of Housing and Urban Development (HUD). (2014). Third allocation, waivers, and alternative requirements for grantees receiving Community Development Block Grant (CDBG) Disaster Recovery Funds in response to Hurricane Sandy. Federal Register, 79(200), 62182-62194. Washington, DC: HUD. Repéré à www.govinfo.gov/content/pkg/FR-201410-16/pdf/2014-24662.pdf

Flint, A. (2011). Wrestling with Moses: How Jane Jacobs took on New York's master builder and transformed the American city. New York, NY: Random House Trade Paperbacks.

Grillo, L. (2019, 23 janvier). East Side Coastal Resiliency hearing (conférence de presse). New York, NY: Department of Design and Construction. 
Hanania, J. (2019, 18 janvier). To save East River Park, the City intends to bury it, New York. Times.

Repéré à www.nytimes.com/2019/01/18/nyregion/to-save-east-river-park-the-city-intends-to-bury-it.html

NYC Mayor Office of Recovery and Resiliency. (2017, 20 janvier). East Side Coastal Resiliency Project. New York, NY: CB3/CB6 Joint Waterfront Task Force (atelier).

NYC Mayor Office of Recovery and Resiliency (2018a, 27 mars). East Side Coastal Resiliency Preliminary Design Update (atelier). New York, NY.

NYC Mayor Office of Recovery and Resiliency (2018b, 11 octobre). East Side Coastal Resiliency Project (atelier). New York, NY.

Ovink, H. et Boeijenga, J. (2018). Too big: Rebuild by Design - A transformative approach to climate change. Rotterdam, Pays-Bas: Nai010 Publishers.

Rebuild by Design. (2015). Rebuild by Design. New York, NY: Fergus.

Rebuild by Design. (2019). Inspiring cities around the world. Repéré à www.rebuildbydesign.org/our-work/city-initiatives

Silver, M. J. (2019, 23 janvier). Oversight: East Side Coastal Resiliency Project (conférence de presse). New York, NY: Committee on Environmental Protection/Committee on Parks and Recreation.

Urban Institute. (2014). The evaluation of the design competition of Rebuild by Design: An initiative of president Obama's Hurricane Sandy rebuilding task. force and the U.S. Department of Housing and Development (rapport officiel). New York, NY: Urban Institute.

Wainwright, O. (2015, 9 mars). Bjarke Ingels on the New York Dryline: «We think of it as the love-child of Robert Moses and Jane Jacobs », The Guardian. Repéré à www.theguardian.com/cities/2015/mar/09/bjarke-ingels-new-york-dryline-parkflood-hurricane-sandy

Wainwright, O. (2017, 30 avril). Street fighter: How Jane Jacobs saved New York from Bulldozer Bob, The Guardian. Repéré à www.theguardian.com/artanddesign/2017/apr/30/citizen-jane-jacobs-the-woman-who-saved-manhattan-fromthe-bulldozer-documentary 